\title{
Study of disturbance wave development in downwards annular flows with a moving frame-of-reference brightness-based laser-induced fluorescence method
}

\author{
Jae Sik An ${ }^{1} \cdot$ Andrey V. Cherdantsev $^{1,2,3} \cdot$ Ivan Zadrazil $^{1} \cdot$ Christos N. Markides $^{1,2}$ (D) \\ Received: 6 January 2020 / Revised: 9 March 2020 / Accepted: 23 June 2020 / Published online: 6 July 2020 \\ (c) The Author(s) 2020
}

\begin{abstract}
A novel moving frame-of-reference brightness-based laser-induced fluorescence (MFoR-BBLIF) method was developed and demonstrated in downwards co-current air-water annular flows. The method was applied to study the downstream development of individual disturbance waves in flows over a range of conditions $\left(\operatorname{Re}_{\mathrm{L}}=276-1321, \mathrm{Re}_{\mathrm{G}}=39,500-79,000\right)$. In this method, the optical measurement system, and hence, the region of interrogation (ROI) was translated physically along the length of the test-section with a velocity close to that of individual disturbance waves to obtain the velocities of individual disturbance-waves as a function of downstream distance from the inlet. It was found that the velocities of individual disturbance waves increase with both downstream distance and gas-liquid flow conditions. In addition, the variation in the wave velocities was more significant at higher gas and liquid Reynolds numbers. The approach can be integrated with many other contactless measurement methods, and can also be used over a range of translation speeds (not necessarily in a "Lagrangian" manner) to study the evolution of important advecting flow phenomena.
\end{abstract}

Christos N. Markides

c.markides@imperial.ac.uk

1 Clean Energy Processes (CEP) Laboratory, Department of Chemical Engineering, Imperial College London, South Kensington Campus, London SW7 2AZ, UK

2 Kutateladze Institute of Thermophysics, Novosibirsk, Russian Federation

3 Novosibirsk State University, Novosibirsk, Russian Federation 


\section{Graphic abstract}

(a)

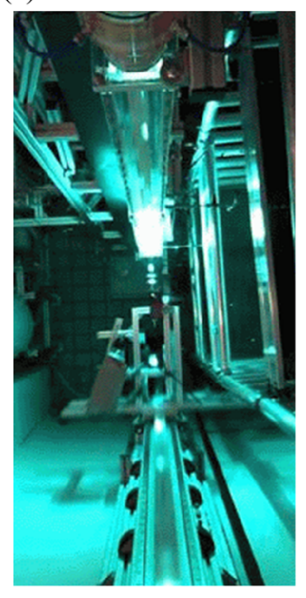

(d)

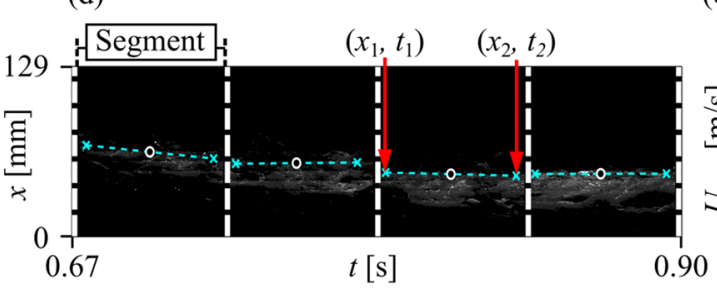

(b)

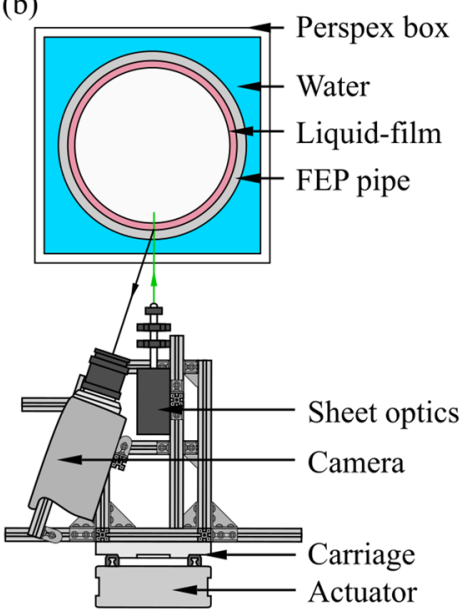

(c)

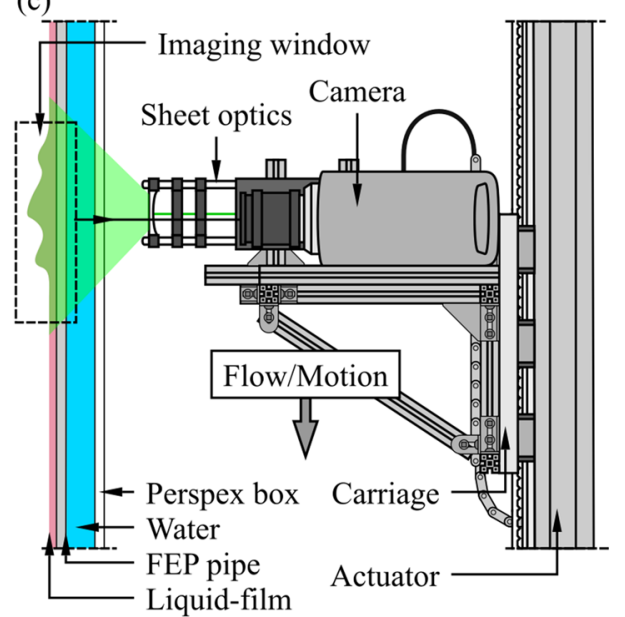

(e)

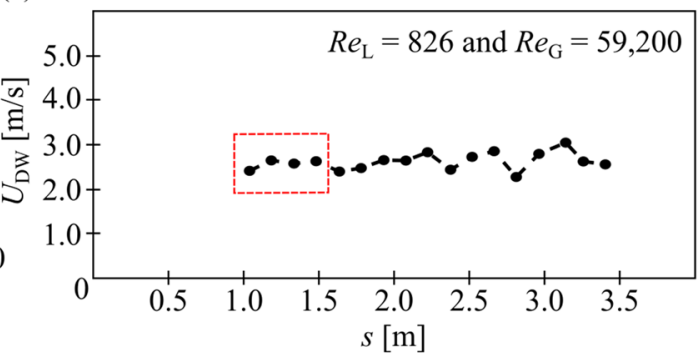

\section{Introduction}

In downwards co-current gas-liquid annular flows in vertical pipes, the gas-phase flows through the centre of the pipe as a continuous gas core, and the liquid-phase flows on the inner surface of the pipe wall as a continuous liquid-film with interfacial waves of varying length and time scales. By convention, the waves are referred to 'ripple waves', which are small amplitude omnipresent waves with short life-spans, and 'disturbance waves', which are large amplitude waves with circumferential coherence (Zhao et al. 2013) and high propagation speeds (Chu and Dukler 1975). Disturbance waves are considered an important interfacial phenomena in annular flows, since these waves carry considerable liquid mass and are a major source of liquid droplet entrainment.

In general, the liquid-film topology has a major effect on the heat and mass transfer properties of many industrial processes, and hence, significant effort has been devoted to measure and understand the characteristics of liquid-films (e.g. mean film thickness, wave frequency, and wave velocity). In several studies, the development of liquid-film characteristics with downstream distance from the liquid inlet was examined through the employment of multiple fixed/ static regions of interrogation (ROI) along the channel with the use of: (i) conductance probes (Wolf et al. 2001; Zhao et al. 2013); (ii) high-speed visualization (Hall Taylor et al. 1963); and (iii) laser-focus displacement (Hazuku et al. 2008). In laser-induced fluorescence (LIF)-based optical methods, such as brightness-based LIF (BBLIF) and planar LIF (PLIF) (see, e.g. Zadrazil et al. 2014; Alekseenko et al. 2015; Cherdantsev et al. 2019), a length of the liquid-film region in the static ROI can be interrogated simultaneously. However, this is dependent on the size of the imaging window, which is normally limited to a few centimetres.

The characteristic limitations of static ROIs in conventional measurements of disturbance waves are the loss of information before-and-after the ROI position, and the short interrogation time. In this work, the authors present a novel technique for conducting dynamic/moving ROI measurements, which we refer to as the moving frame-of-reference brightness-based laser-induced fluorescence (MFoR-BBLIF) method. This method overcomes the limitations associated with the use of static ROIs, and allows investigations of disturbance wave development over long axial lengths 
and interrogation times. In MFoR-BBLIF, the ROI position moves physically along the channel, thus significantly increasing the interrogation time of individual disturbance waves and recovering key wave characteristics (e.g. disturbance wave velocities) as a function of downstream distance from the channel inlet.

\section{Experimental method}

The Downwards Annular Flow Observation Facility (DAFLOF) was used to generate downwards co-current air-water annular flows in a vertical test-section (for details see Cherdantsev et al. 2019), while a linear actuator system (ROLLON) was employed to translate the MFoR-BBLIF optical measurement system, and hence, the dynamic ROI along the test-section. The linear deviation of the alignment between the test-section $(D=32.4 \mathrm{~mm}, L=4.0 \mathrm{~m})$ and actuator was estimated to be $\pm 1 \mathrm{~mm}$. The relevant measurement optics were secured onto the carriage of the linear actuator, which was belt-driven and powered by a $1.5-\mathrm{kW}$ motor (MAC1500, JVL Industri Elektronik A/S). The motor was controlled by dedicated software provided by the supplier
(MacTalk). The maximum available linear motion of the carriage was $5.0 \mathrm{~m}$ with a repeatability accuracy of $\pm 0.05 \mathrm{~mm}$ (stated by the supplier), and covered the entire test-section length. Prior to the measurements, a set of calibration exercises were performed to obtain the velocity profile of the carriage for a range of motor speeds at a constant acceleration and deceleration. For these exercises, distinct markers were attached along the edge of the test-section with equal spacing, and instantaneous images of the markers were collected for a given motor speed. The calibration images were processed to recover the carriage velocity as a function of downstream distance and time.

The MFoR-BBLIF optical arrangement on the actuator carriage is shown in Fig. 1b, c. The liquid-phase was seeded with Rhodamine $6 \mathrm{G}$ dye (dye concentration $c \approx 4 \mathrm{mg} / \mathrm{L}$ ) and excited by a laser-sheet (wavelength $\lambda=510.6 \mathrm{~nm}$; pulse frequency $f_{\text {pulse }}=10 \mathrm{kHz}$; sheet thickness $\tau \approx 200 \mu \mathrm{m}$ ), which was produced with dedicated sheet-optics connected to a $\mathrm{Cu}$-vapour laser system (LS20-10, Oxford Lasers) via a fibre-optic cable. The fluorescence signals were collected by a high-speed camera (i-SPEED 3, i-X Cameras, $f_{\text {camera }}=10 \mathrm{kHz}$ ) equipped with a $540-\mathrm{nm}$ long-pass optical filter. The camera was positioned at an angle of $\approx 10^{\circ}$ to (a)

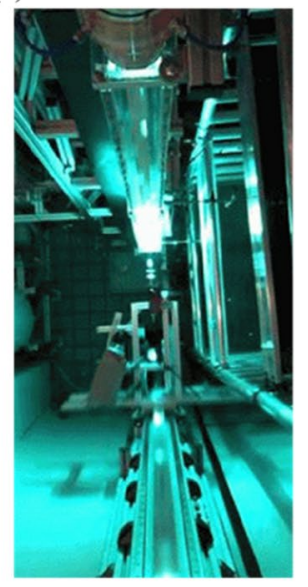

(d)

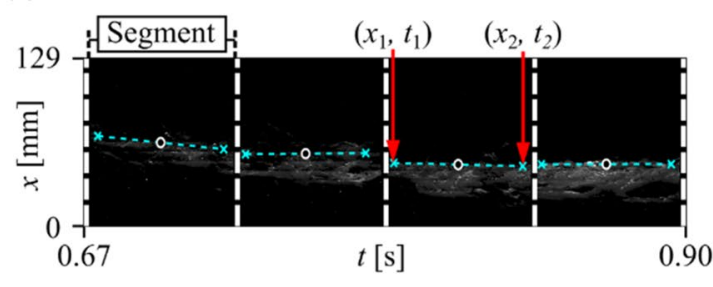

(b)

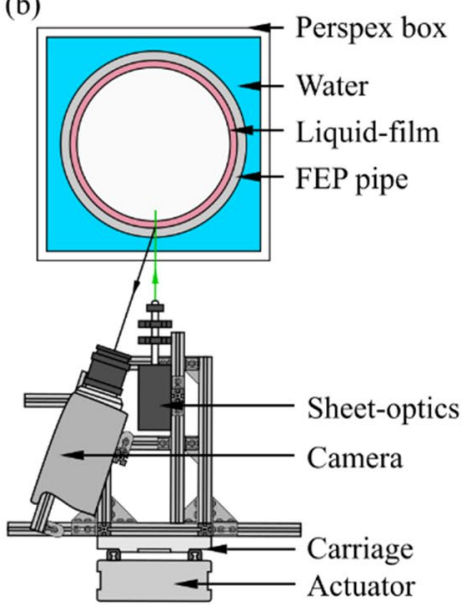

(c)

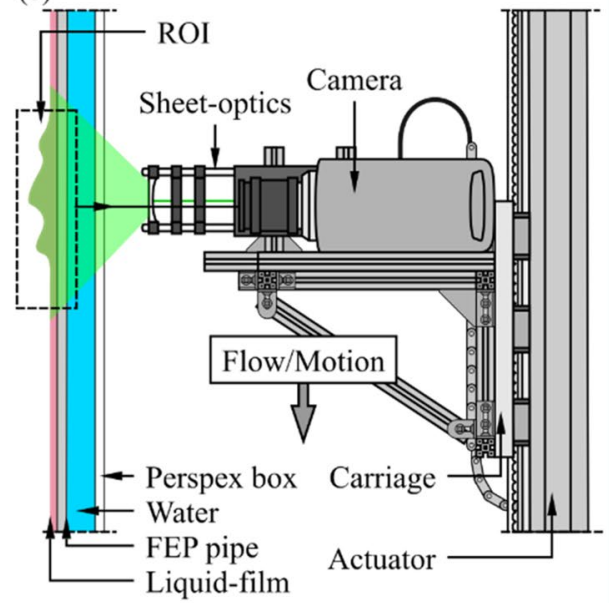

(e)

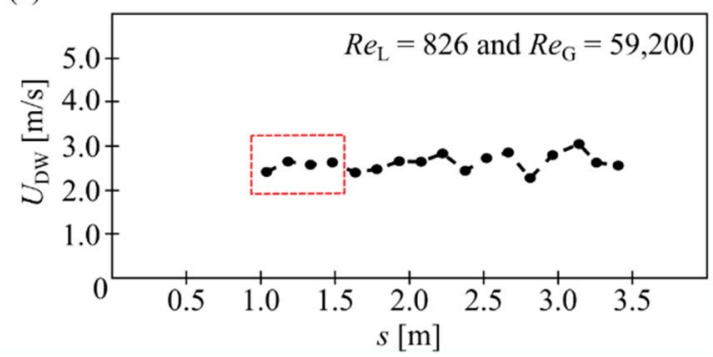

Fig. 1 Arrangement of the MFoR-BBLIF optics: a photograph from the top of the facility; $\mathbf{b}$ top-view; and (c) side-view. For a downwards annular flow at $\operatorname{Re}_{\mathrm{L}}=826$ and $\operatorname{Re}_{\mathrm{G}}=59,200$ : $\mathbf{d}$ front-slope identifica-

tion of a single disturbance wave in a sample $x-t$ matrix; and e $U_{\mathrm{DW}}$ vs $s$ of the disturbance wave in (d) 
the excitation plane. The size of the imaging window was $129 \mathrm{~mm} \times 32 \mathrm{~mm}$, and the spatial resolution was $245 \mu \mathrm{m} /$ pixel. In each experimental run for a given flow condition, the optical measurement system and ROI moved from topto-bottom of the test-section, and the parameters set on the motor were based on the disturbance wave velocity data obtained by Webb and Hewitt (1975). For each flow condition, $\approx 10-12$ runs were performed. The investigated gas and liquid Reynolds numbers were $\operatorname{Re}_{\mathrm{L}}=Q_{\mathrm{L}} / \pi D \nu_{\mathrm{L}}=276-1,321$ and $\mathrm{Re}_{\mathrm{G}}=j_{\mathrm{G}} D / \nu_{\mathrm{G}}=39,500-79,000$, respectively.

The collected images were processed to identify individual disturbance waves along the entire length of the test-section and to extract their velocities. For each run, the images were converted into a $x-t$ (spatiotemporal) matrix of film thickness (see, e.g. Fig. 2a). Each row of a $x-t$ matrix consists of local instantaneous fluorescence intensity values along the central-line of the laser-sheet on a corresponding frame. The fluorescence was then converted into local instantaneous values of film thickness in accordance with standard BBLIF procedures using a record of a flow at $\mathrm{Re}_{\mathrm{L}}=150$ and $\mathrm{Re}_{\mathrm{G}}=0$ as a reference signal (for details, see Cherdantsev et al. 2019). In Fig. 2b, examples of instantaneous film thickness profiles along $x$ are shown for 5 time instants marked in Fig. 2a.

Black-regions were observed in the $x-t$ matrix due to the de-synchronization of the camera and the laser, which lead to a natural division of the $x-t$ matrix into even segments. In each segment with an identified disturbance wave, the locations $\left(x_{1}, t_{1}\right)$ and $\left(x_{2}, t_{2}\right)$ of the front-slope of the wave were determined as shown in Fig. 1d. The gradient between the two points corresponds to the relative velocity of the disturbance wave in the moving system, $U_{\mathrm{DW}-\mathrm{R}}$ at time, $t_{\mathrm{av}}$. Therefore, a negative gradient indicated a
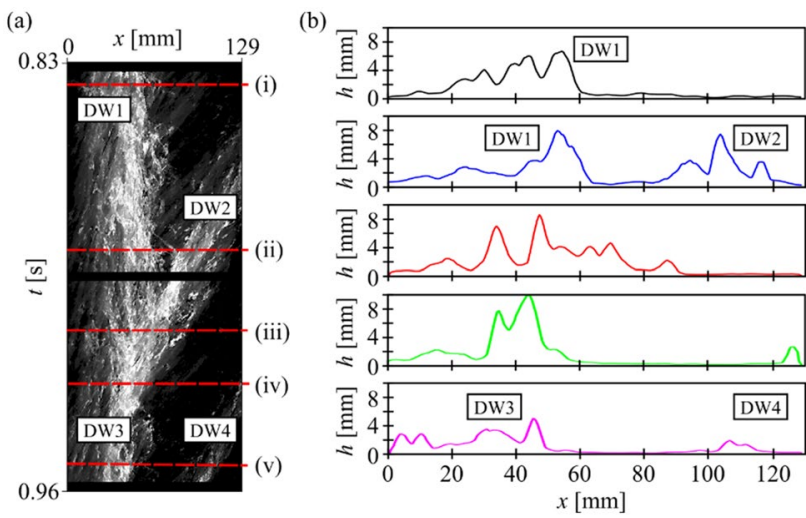

Fig. 2 Disturbance wave coalescence at $\operatorname{Re}_{\mathrm{L}}=1321$ and $\operatorname{Re}_{\mathrm{G}}=79,000$ : a raw fluorescence intensity signals in the form of a $x-t$ matrix; and b instantaneous film-thickness profiles for instants (i) to (v) with an incremental shift of $4 \mathrm{~mm}$. The profiles are smoothed using median and running-average filters with 15 -pixel $(\approx 3.6 \mathrm{~mm})$ windows disturbance wave moving slower than the camera, and vice versa. The disturbance wave velocity, $U_{\mathrm{DW}}$ at $t_{\mathrm{av}}$ is defined as $U_{\mathrm{DW}}=U_{\mathrm{AC}}+U_{\mathrm{DWR}}$. Since the movement parameters of the optics is known, the downstream position of the disturbance wave from the gas-liquid inlet, $s$ at $t_{\text {av }}$ can also be calculated. The entire $x-t$ matrix is examined to identify whether the disturbance wave in consecutive segments are identical or separate. In Fig. 1d, the dynamic ROI moves with a disturbance wave $\left(\operatorname{Re}_{\mathrm{L}}=826, \operatorname{Re}_{\mathrm{G}}=59,200\right)$ for $t=0.23 \mathrm{~s}$ and $s=0.44 \mathrm{~m}$. A plot of $U_{\mathrm{DW}} \mathrm{vs} s$ for this wave is given in Fig. 1e.

\section{Results and discussion}

Disturbance wave coalescence as described by Wilkes et al. (1983) was one of many phenomena observed in the qualitative analysis of the instantaneous MFoR-BBLIF images. An example is shown in the form of a raw $x-t$ matrix and a sequence of instantaneous $h(x)$ profiles in Fig. 2a and b, respectively. The time duration between Frames (i) to (v) was $0.13 \mathrm{~s}$, which corresponds to a downstream shift of DW1 by $0.43 \mathrm{~m}$. The disturbance wave, DW1 approaches a second disturbance wave, DW2 further downstream as the velocity of DW1 is greater than DW2. The waves coalesce and the resultant wave, DW3 moves with approximately the same velocity as DW1. It is interesting that at the exact moment of coalescence [time instant (iv), green-line in Fig. 2b] a very large (i.e. high) 'hump' is formed at the front region of DW1, which disappears later [time instant (v), purpleline], probably due to both significant entrainment and more intense generation of slow ripples.

For the range of investigated flow conditions, the velocity of individual disturbance waves as a function of downstream distance from the test section inlet is shown in Fig. 3a-i. Since the main objective of the implementation of this technique was to continuously observe individual waves with downstream distance and extended time durations, the number of individual disturbance waves were deemed insufficient for statistical analysis of the data. However, there are a number of noticeable trends that can be observed within the plots.

Along the entire length of the test section, the disturbance wave velocities increase with $\operatorname{Re}_{\mathrm{G}}$ and $\mathrm{Re}_{\mathrm{L}}$, however, the influence of the $\operatorname{Re}_{\mathrm{G}}$ seems to be greater than $\operatorname{Re}_{\mathrm{L}}$. For the given flow conditions, $U_{\mathrm{DW}}$ increases with $s$. Significant individual acceleration of disturbance waves at the initial stages of their development was observed in agreement to Alekseenko et al. (2015). Similar to the observations made by Wolf et al. (2001) in upwards annular flow; $U_{\mathrm{DW}}$ appears to reach a near-constant value as the wave approaches a downstream distance of $3.5 \mathrm{~m}$. The variation in the velocities of individual disturbance waves is more significant at 


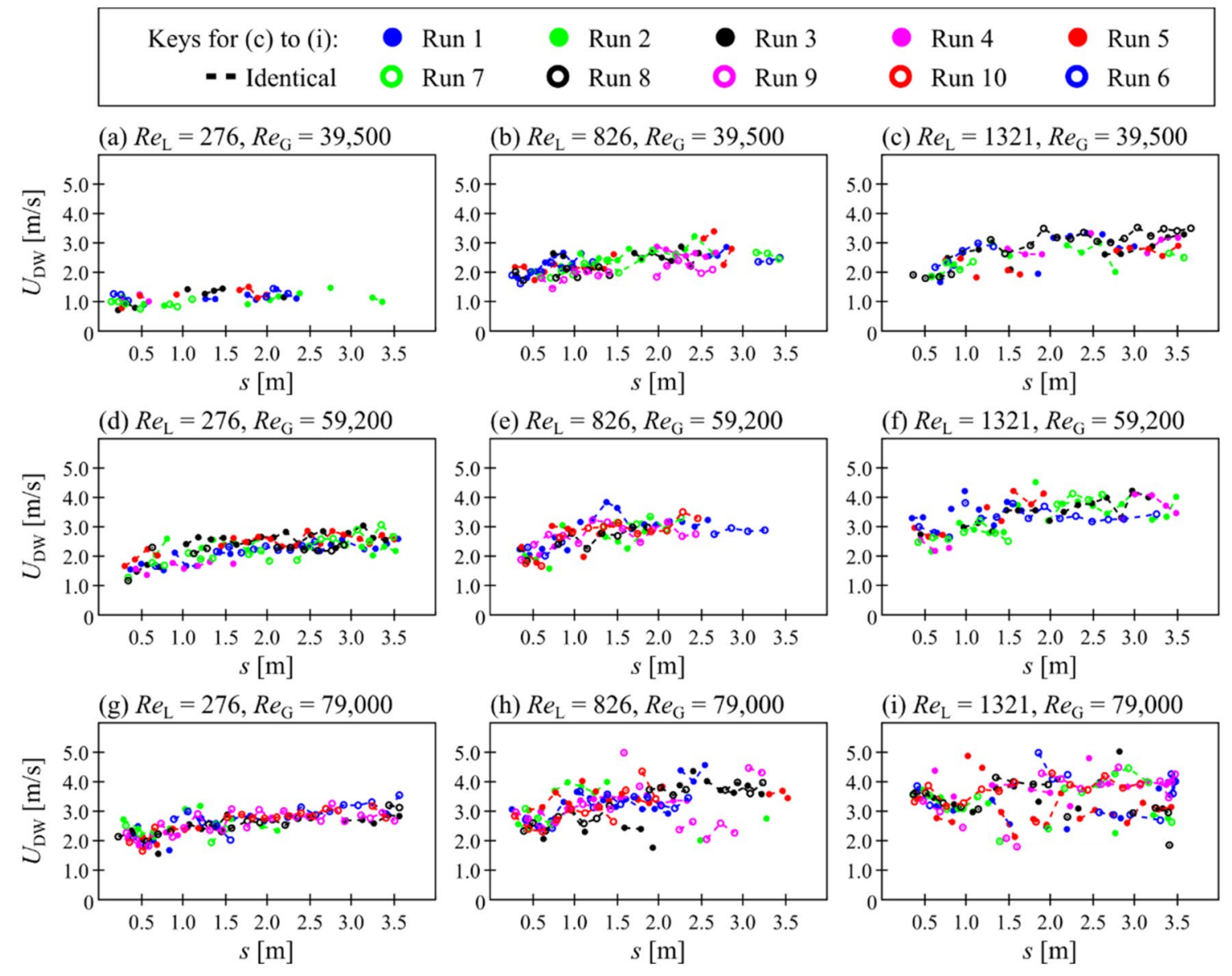

Fig. 3 For the range of investigated flow conditions, individual disturbance wave velocities $\left(U_{\mathrm{DW}}\right)$ as a function of downstream distance from the channel inlet $(s)$

larger gas and liquid flow-rates. The investigated flow conditions are in the 'regular wave' regime (see Webb and Hewitt 1975). The gas-liquid interface is highly agitated and the rate of liquid droplet entrainment is very high, which could cause pressure gradient fluctuations along the axial length of the channel.

\section{Conclusion}

A new experimental method, which is referred to as moving frame-of-reference brightness-based laser-induced fluorescence (MFoR-BBLIF), has been reported in this work. This technique can overcome the limitations of conventional static ROI measurement approaches and has the capability of conducting dynamic measurements of the development of disturbance waves from the flow inlet over long lengths and interrogation times. The ROI in this technique moves along the channel to extend the time duration of individual disturbance waves within the interrogation window and continuously recover the wave characteristics (e.g. wave velocity) as disturbance waves travel downstream. The MFoR-BBLIF approach was demonstrated on disturbance waves in downwards air-water annular flows $\left(\mathrm{Re}_{\mathrm{L}}=276-1321\right.$, $\left.\operatorname{Re}_{\mathrm{G}}=39,500-79,000\right)$. Individual disturbance wave velocities increase with downstream distance and gas-liquid flow conditions. In addition, the variation in the individual velocities increase with $\operatorname{Re}_{\mathrm{L}}$ and $\mathrm{Re}_{\mathrm{G}}$. Future work will be aimed at the recovery of other wave characteristics as single waves propagate downstream, as well as, a systematic study of disturbance wave coalescence and generation of fast and slow ripples by individual disturbance waves.

The moving-frame-of-reference (MFoR) approach presented herein has a wider range of research applications than that considered in this paper: (i) the MFoR approach can be integrated with other contactless, non-intrusive methods, such as high-speed visualization or PLIF with structured illumination (S-PLIF); (ii) MFoR can be used to study the downstream evolution of other individual interfacial phenomena with large development lengths in different multiphase flows (e.g. bubbly flow, slug flow, stratified flow, etc.), and also single-phase turbulent flows (i.e. with the 
application of PIV, temperature/concentration sensitive LIF, background oriented Schlieren (BOS), etc.); and (iii) it is not necessary to follow a particular interfacial structure in a "Lagrangian" manner as demonstrated in this paper, for example, it is possible to utilize a relatively low dynamic ROI speed to study the downstream evolution of flow characteristics in an "Eulerian" (but still dynamic) approach.

Acknowledgements The work was supported by Russian Government "Megagrant" project 075-15-2019-1888, the UK Department for International Development (DFID) through the Royal Society-DFID Africa Capacity Building Initiative, and the UK Engineering and Physical Sciences Research Council (EPSRC) [grant numbers EP/K008595/1, and EP/L020564/1]. Data supporting this publication can be obtained on request from cep-lab@imperial.ac.uk.

Open Access This article is licensed under a Creative Commons Attribution 4.0 International License, which permits use, sharing, adaptation, distribution and reproduction in any medium or format, as long as you give appropriate credit to the original author(s) and the source, provide a link to the Creative Commons licence, and indicate if changes were made. The images or other third party material in this article are included in the article's Creative Commons licence, unless indicated otherwise in a credit line to the material. If material is not included in the article's Creative Commons licence and your intended use is not permitted by statutory regulation or exceeds the permitted use, you will need to obtain permission directly from the copyright holder. To view a copy of this licence, visit http://creativecommons.org/licenses/by/4.0/.

\section{References}

Alekseenko SV, Cherdantsev AV, Cherdantsev MV, Isaenkov SV, Markovich DM (2015) Study of formation and development of disturbance waves in annular gas-liquid flow. Int J Multiph Flow 77:65-75

Cherdantsev AV, An JS, Charogiannis A, Markides CN (2019) Simultaneous application of two laser-induced fluorescence approaches for film thickness measurements in annular gas-liquid flows. Int $\mathrm{J}$ Multiph Flow 119:237-258

Chu KJ, Dukler AE (1975) Statistical characteristics of thin, wavy films III. Structure of the large waves and their resistance to gas flow. AIChE J 21:583-593

Hall Taylor N, Hewitt GF, Lacey PMC (1963) The motion and frequency of large disturbance waves in annular two-phase flow of air-water mixtures. Chem Eng Sci 18(8):537-552

Hazuku T, Takamasa T, Matsumoto Y (2008) Experimental study on axial development of liquid film in vertical upward annular twophase flow. Int J Multiph Flow 34(2):111-127

Webb DR, Hewitt GF (1975) Downwards co-current annular flow. Int J Multiph Flow 2:35-49

Wilkes NS, Azzopardi BJ, Thompson CP (1983) Wave coalescence and entrainment in vertical annular two-phase flow. Int J Multiph Flow 9:383-398

Wolf A, Jayanti S, Hewitt GF (2001) Flow development in vertical annular flow. Chem Eng Sci 56:3221-3235

Zadrazil I, Matar OK, Markides CN (2014) An experimental characterization of downwards gas-liquid annular flow by laser-induced fluorescence: flow regimes and film statistics. Int J Multiph Flow 60:87-102

Zhao Y, Markides CN, Matar OK, Hewitt GF (2013) Disturbance wave development in two-phase gas-liquid upwards vertical annular flow. Int J Multiph Flow 55:111-129 